\title{
EQUAÇÃO DE VIABILIDADE PARA DETERMINAR A LONGEVIDADE DE SEMENTES DE TRIGO SOB CONDIÇÕES DIFERENCIADAS EM ARMAZENAMENTO CONVENCIONAL ${ }^{1}$
}

\author{
MARIA CELESTEMARCONDES²; CLAUDINEI ANDREOLI ${ }^{3}$;EDISON MIGLIORANZA ${ }^{4}$
}

\begin{abstract}
RESUMO - O objetivo deste trabalho foi aplicar a equação simplificada de viabilidade desenvolvida por Andreoli (1998) para predizer a longevidade de sementes de trigo das cultivares BRS 210, BRS 208, CD 104, IAPAR 78 e IPR 85, em condições de armazenamento convencional, nos municípios de Mauá da Serra e Londrina, PR. A equação simplificada é dada pelo modelo, $\mathrm{Vp}=\mathrm{Vi}-(\operatorname{tg} \beta)$. p, em que Vp é a viabilidade em 'probit' no período $\mathrm{p}$, Vi é a germinação inicial do lote, e $\operatorname{tg} \beta$ é a taxa de deterioração da semente para cada cultivar. Os lotes de sementes escolhidos ao acaso foram divididos em quatro partes, embalados em sacaria de polipropileno trançado e armazenados por 300 dias em armazéns convencionais nos dois municípios. Os dados de germinação foram transformados em 'probit' e a declividade da reta $(\operatorname{tg} \beta)$ foi calculada entre zero e 30 dias. As taxas de deterioração variaram de $0,4 \times 10^{-3}$ a $1,3 \times 10^{-3}$, sendo que nas condições de Londrina as taxas foram maiores, indicando que a germinação da semente declinou mais rapidamente do que em Mauá da Serra. O modelo prediz com acurácia a longevidade das sementes de trigo, independente do genótipo, em armazém convencional. A germinação inicial das sementes $\left(\mathrm{V}_{1}\right)$ não afetou a taxa de deterioração.

Termos para indexação: Triticum aestivum, semente, germinação, deterioração
\end{abstract}

\section{VIABILITY EQUATION TO DETERMINE SEED LONGEVITY OF WHEAT UNDER DIFFERENTIATED CONDITIONS DURING OPEN WAREHOUSE STORAGE}

\begin{abstract}
The objective of this work was to apply the simplified equation of viability developed by Andreoli (1998), to predict changes in seeds viability during open warehouse storage. Seeds of wheat cultivars BRS 210, BRS 208, CD 104, IAPAR 78 and IPR 85 were packed in raffia bags and stored for 0, 30, 60, 90, 120, 180, 240, and 300 days in an open warehouse at two locations: Mauá da Serra and Londrina; both in Paraná State, Brazil. The simplified equation is given by the model $\mathrm{Vp}=\mathrm{Vi}-(\operatorname{tg} ß) . \mathrm{p}$, in which $\mathrm{Vp}$ is seed viability in probit for a period $\mathrm{p}$ (days), $\mathrm{Vi}$ is the initial seed viability in probit and tg $\beta$ is the seed deterioration rate for wheat at determined storage conditions. The germination data was transformed in probit and the deterioration rate $(\operatorname{tg} \beta)$ between 0 and 30 days was calculated.The coefficient $\operatorname{tg} \beta$ ranged from $0,4 \times 10^{-3}$ to $1,3 \times 10^{-3}$, depending on locations and cultivars. In Londrina, the coefficient tg $\beta$ was higher, indicating that the germination declined faster than Mauá da Serra. This model has accurately predicted wheat seeds germination, independently of the genotype, under open storage. The initial quality of the seed $\left(\mathrm{V}_{1}\right)$ did not affect the seed deterioration rate.
\end{abstract}

Index terms: Triticum aestivum L., seed, germination, vigor, aging.

\footnotetext{
1 Submetido em 08/11/2005. Aceito para publicação em 20/07/2007. Parte da Dissertação de Mestrado do primeiro autor apresentado na Universidade Estadual de Londrina.

1 Eng $^{\mathrm{a}} \mathrm{Agr}^{\mathrm{a}}$, doutoranda em Agronomia, (UEL) - Universidade Estadual de Londrina, PR, email: celeste@ seab.pr.gov.br

2 Eng $^{\mathrm{a}}$ Agr ${ }^{\mathrm{a}}$ Dr. Pesquisador, Embrapa Soja, Londrina, PR., email: andreoli@ cnpso.embrapa.br.

3 Eng $^{\circ}$ Agr $^{\circ}$ Dr., professor do Departamento de Agronomia, (UEL) - Universidade Estadual de Londrina, PR, email: emiglior@uel.br
} 


\section{INTRODUÇÃO}

O potencial de conservação das sementes é determinado pela velocidade do processo de deterioração e pode ser variável entre diferentes lotes da mesma espécie e mesma cultivar, armazenados sob as mesmas condições (Delouche e Baskin, 1973). Fatores como temperatura, umidade relativa do ar e a qualidade fisiológica inicial das sementes influenciam a perda de vigor no armazenamento (Roberts, 1960, 1972, 1986).

Diferentes modelos matemáticos foram desenvolvidos para predizer a perda de qualidade da semente durante o armazenamento e determinar a taxa de deterioração de várias espécies (Roberts, 1960, 1961, 1973; Ellis e Roberts, 1980, 1981; Ellis, 1988; Ellis et al, 1990; Tekrony et al., 1993; Fabrizius et al., 1999; Tang et al., 1999, 2000; Andreoli, 1998, 2004).

Roberts $(1960,1961)$ para predizer a longevidade das sementes de trigo e arroz armazenadas em condições controladas de temperatura e umidade, descreveu uma equação básica de viabilidade, $\mathrm{v}=\mathrm{Ki}-\mathrm{p} / 10^{\mathrm{KL}-\mathrm{Clm}-\mathrm{C} 2 \mathrm{t}}$. Com o intuito de melhorar a confiabilidade dessa equação, Ellis e Roberts (1980) propuseram a versão melhorada (2), a seguir:

$\mathrm{V}=\mathrm{Ki}-\mathrm{p} / 10_{\mathrm{e}}^{\mathrm{K}-\mathrm{C}} \underset{\mathrm{w}}{\log \mathrm{m}-\mathrm{C} t-\mathrm{C} t 2} \mathrm{Q}$

onde $v$ é a viabilidade de semente em 'probit' em qualquer período $(p)$, para a combinação de temperatura $(t) \mathrm{e}$ umidade $(m)$; as constantes, $\mathrm{K}_{\mathrm{e}} \cdot \mathrm{C}_{\mathrm{w}}, \mathrm{C}_{\mathrm{H}}$ e $\mathrm{C}_{\mathrm{Q}}$ são especificas para cada espécie, independente do genótipo e da qualidade inicial da semente; $K i$ é a qualidade inicial e específica do lote de semente. Na prática, a equação 2 exige cálculos matemáticos difíceis e a determinação das constantes exige experimentos complexos e de longa duração. A equação de viabilidade (equação 1]) é composta de duas equações separadas. A primeira (Equação 2) descreve a curva de sobrevivência da semente em termos da viabilidade (v. probit porcentagem de viabilidade) esperada após um período de armazenamento (p. dias).

$$
\mathrm{v}=\mathrm{Ki}-\mathrm{p} / \sigma
$$

A suposição é que as diferenças de qualidade entre lotes de semente não afeta $\sigma$ (o desvio padrão da freqüência da distribuição da curva de sobrevivência), mas considera $K i$. Ao contrário, as condições do armazenamento não tem efeito em $K i$, e afetam somente $\sigma$. A relação entre $\sigma$ e as constantes das condições ambientais é descrita pela equação 3 .

$\log \sigma=\mathrm{K}_{\mathrm{E}}-\mathrm{C}_{\mathrm{w}} \log \mathrm{m}-\mathrm{C}_{\mathrm{h}} \mathrm{t}-\mathrm{C}_{\mathrm{Q}} \mathrm{t}^{2}$

O $\sigma$ pode ser percebido como também o tempo requerido para a viabilidade cair uma unidade de probit (de 97,7 $84,1 \%$ ou $84,1-50 \%$ ). Assim, é uma medida da longevidade da semente. O mais importante pré-requisito para uso da equação 1 é a premissa da mesma taxa de deterioração entre lotes de uma mesma espécie armazenados numa condição ambiental idêntica. Se essa suposição for válida, as constantes da equação 3 podem ser determinadas, do contrário, elas não são universais para todos lotes de sementes (Ellis e Roberts, 1980, 1981; Roberts e Ellis, 1989; Tang et al., 1999).

Andreoli (1998, 2004), identificando a necessidade de predição exata e precisa da deterioração de sementes em condições de armazenamento e aliando as dificuldades acima da equação equação 1 , simplificou a equação básica de viabilidade de Ellis e Roberts (1980) para predizer as mudanças na germinação e longevidade das sementes durante o período de armazenamento convencional. Este modelo estimou com alta confiabilidade $\left(r^{2}>0,95\right)$ a viabilidade das sementes de soja e milho nas condições de Sete Lagoas, MG. e Brasília, DF.

O objetivo deste trabalho foi testar a equação simplificada de viabilidade, preconizada por Andreoli (1998), para predizer as mudanças na germinação e na taxa de deterioração de diversos lotes de sementes de trigo, armazenadas sob condições de armazenamento convencional, em duas localidades no estado do Paraná, Mauá da Serra e Londrina.

\section{MATERIAL E MÉTODOS}

Equação simplificada -A relação entre a temperatura, umidade da semente e o período médio de viabilidade das 
sementes de várias espécies foram proposta por Roberts (1960, 1961, 1972). Assim, a perda de viabilidade definida pelas três equações básicas de Roberts (1972) tem as seguintes premissas:

1) A freqüência de sementes mortas num determinado período, em uma população de sementes, armazenada em condições constantes, é descrita pela Equação [4] da distribuição normal:

$$
\underset{2 \sigma 2}{(p-\mu) 2} \quad y=\frac{1}{\sigma \sqrt{ } 2 \text { II }} e
$$

em que y é a freqüência relativa de sementes mortas no tempo $p, \mu$ é o período de viabilidade média $\left(\mathrm{p}_{50}\right)$, e $\sigma$ é o desvio padrão da distribuição das sementes mortas no tempo.

2) A dispersão da distribuição no tempo é proporcional ao período de viabilidade média, i.e.

$$
\sigma=\mathrm{K}_{\sigma \cdot \mathrm{p}_{50}}
$$

em que $\mathrm{K}_{\sigma}$ é uma constante das espécies e $\mathrm{p}_{50}$ é o período de viabilidade média.

3) A relação entre a temperatura, o teor de umidade da semente e o período de viabilidade médio são descritos pela seguinte equação:

$$
\log \mathrm{p}_{50}=\mathrm{K}_{\mathrm{v}}-\mathrm{C}_{1} \mathrm{~m}-\mathrm{C}_{2} \mathrm{t}
$$

em que $\mathrm{m}$ é a umidade da semente (por cento, em base úmida), t é a temperatura constante $\left({ }^{\circ} \mathrm{C}\right)$ e $\mathrm{K}_{\mathrm{v}}, \mathrm{C}_{1}$ e $\mathrm{C}_{2}$ são constantes.

Conforme a equação 4 , as curvas de sobrevivência da semente são retas de coeficiente de regressão, quando a porcentagem de viabilidade é transformada em 'probit'. Nessas retas, o coeficiente de regressão é dado por $1 / \sigma$ (Finney, 1971; Ellis e Roberts, 1980), em que $\sigma$ é o desvio padrão da distribuição de sementes mortas no tempo. Assim, a equação 7 de viabilidade pode ser descrita como:

$$
\mathrm{v}=\mathrm{Ki}-(\underline{1 / \sigma)} \cdot \mathrm{p},
$$

em que $v$ é a germinação em 'probit' no tempo $p, \mathrm{e} \mathrm{Ki}=\mathrm{v}$ quando $\mathrm{p}=0$.
A taxa de deterioração da semente em armazenamento convencional foi descrito por Andreoli (2004), conforme equação 8:

$$
\frac{V p-V i}{\mathrm{P}}=-(\operatorname{tg} \sigma)
$$

Portanto, o coeficiente de regressão da equação melhorada [4], $\sigma$, dado pelo termo $10^{\mathrm{Ke}-\mathrm{Cwlogm}-\mathrm{CHt}-\mathrm{CQ} t 2}$ é igual ao coeficiente angular $(\operatorname{tg} \sigma)$ da equação simplificada 8 e, substituindo-o em [7] tem-se a equação simplificada equação 9:

$$
\mathrm{Vp}=\mathrm{Vi}-(\operatorname{tg} \sigma) \cdot \mathrm{p}
$$

em que Vp é a viabilidade das sementes em 'probit' no tempo $p$, Vi é a germinação inicial em 'probit' e tgo é a taxa de deterioração da semente para cada espécie. A constante tgâ é especifica de cada espécie e depende das condições ambientais do armazém, mas independe do genótipo e da qualidade inicial da semente (Roberts e Abdalla, 1968; Roberts, 1973). Vi é especifico para cada lote de semente e é a medida da sua qualidade inicial. $\mathrm{O}$ valor de $(-\operatorname{tg} \sigma)$ é estimado, calculando-se o declínio da viabilidade $(\mathrm{Vp}-\mathrm{Vi})$ por um determinado período $(\Delta \mathrm{p})$, nas condições específicas do armazém.

Desde que Vi seja, de fato, a percentagem de viabilidade em 'probit' no início do armazenamento, há necessidade de se estimar seu valor realizando um teste de germinação no início do armazenamento. Roberts (1986) argumentou que o simples teste de germinação não estima adequadamente a qualidade da semente no início do armazenamento. $\mathrm{O}$ erro de amostragem no teste de germinação com 400 sementes pode ser alto e, assim, o intervalo de confiança desse valor também pode ser elevado e uma pequena diferença na percentagem de germinação para altos valores pode causar grandes efeitos no valor de $\mathrm{Vi}$, e, conseqüentemente, no potencial de longevidade.

Método - Para testar o modelo proposto na Eq. [9], foram selecionados, ao acaso, na empresa produtora Sementes Mauá Ltda, Mauá da Serra, PR, oito lotes de sementes de trigo, formados com 480 sacas de $25 \mathrm{~kg}$ cada, da safra 2003, das cultivares BRS 210, BRS 208, CD 104, 
IAPAR 78 e IPR 85; cultivares estas que representaram quase $60 \%$ do total de semente produzida e plantada no Estado (Paraná, 2003).

Os lotes selecionados foram divididos em quatro partes, ficando duas partes da amostra depositados em armazenagem convencional em Mauá da Serra e duas em Londrina, PR, por um período de 300 dias. Nos intervalos de $0,30,60,90,120,180,240$ e 300 dias, as amostras foram coletados nas duas localidades no mesmo dia, com calador de alumínio, para determinação da umidade e análise de germinação. As amostras foram transportadas para Laboratório de Sementes da Embrapa Soja, em Londrina, PR, em caixas de papel devidamente identificadas. No início do armazenamento, tempo 0 dias (19 de novembro de 2003) a germinação inicial dos lotes (Vi) foi determinada.

Durante o período de armazenagem estabelecido, a temperatura média $\left({ }^{\circ} \mathrm{C}\right)$ e a umidade relativa do ar $(\%)$, foram registradas nas duas localidades por um termohigrógrafo. Comparado aos valores registrados externamente, verificou-se que não houve variação significativa, conforme demonstrado nas Figuras 1 e 2.

Determinações realizadas -As análises foram realizadas no laboratório da Embrapa Soja, Londrina, PR, sendo o grau de umidade das sementes determinado pelo método de estufa, marca Fabbe-Primar, a $105^{\circ} \mathrm{C} \pm 1$, por 24 horas, utilizando-se quatro repetições de $25 \mathrm{~g}$ para cada amostra e os resultados foram expressos em percentagem, conforme recomendações das Regras para Análise de Sementes (Brasil 1992), demonstrados na Figura 3.

A germinação das sementes de trigo para cada amostra foi conduzida utilizando-se 400 sementes distribuídas em oito repetições de 50 sementes, em cada período de armazenamento estabelecido pelo cronograma, semeadas em rolo de papel-toalha marca Germitest, e colocadas em germinador de sementes tipo Mangelsdor - Elo's, regulado para manter a temperatura constante de $20^{\circ} \mathrm{C} \pm 1$. A contagem foi efetuada aos três e cinco dias após a instalação do teste, seguindo-se as recomendações das Regras para Análise de Sementes (Brasil, 1992). Os resultados relativos à avaliação de três dias não foram significativos, por esta razão desconsiderados. Por este estudo, somente as plântulas viáveis e normais após o quinto dia foram testadas no modelo, diferenciando de Ellis e Roberts (1980), que consideraram apenas as sementes mortas .
Análise estatística - Os dados de germinação foram transformados em 'probit' e ajustados pela equação [Vp $=\mathrm{Vi}-(\operatorname{tg} \sigma) \cdot \mathrm{p}]$, para predizer a viabilidade das sementes de trigo durante o armazenamento. Na determinação do coeficiente angular tgâ (taxa de deterioração da semente) em cada localidade, para cada cultivar e lote, utilizou-se a viabilidade inicial e após 30 dias de armazenamento (Vi $\mathrm{V}_{30}$ ). A equação simplificada, proposta por Andreoli (1998, 2004), foi utilizada para predizer a germinação das sementes durante cada período de armazenamento. O modelo estatístico foi blocos inteiramente casualizados com duas repetições.

Os dados de cada localidade, para cada lote e cultivar, foram ajustados pelo modelo da equação simplificada, e os valores de $r^{2}$ entre os valores observados e esperados foram calculados para estimar a confiabilidade do modelo proposto.

\section{RESULTADOS E DISCUSSÃO}

O teor de água, em média, na semente de trigo, durante o período de armazenamento, para todas as cultivares analisadas nos dois municípios manteve-se entre 10,38 e $12,68 \%$. Os teores de água das sementes armazenadas no município de Londrina foram menores do que os de Mauá da Serra; isto se deve as condições climáticas que apresentaram menor umidade relativa em Londrina (Figuras 1 e 2).

A germinação, predita pela equação simplificada Eq.[9], foi comparada com a germinação observada no período de 0 a 300 dias para sementes de trigo, cultivar BRS 210, nas duas localidades, Figura 4. A equação simplificada previu com acurácia a queda de viabilidade dos lotes 6 e 8 no município de Mauá da Serra (Figura 4a) e dos lotes 1, 3 e 8 em Londrina (Figura 4b). A germinação ficou acima de $95 \%$ após 300 dias de armazenamento nas duas localidades. A queda de viabilidade estimada pelo coeficiente angular $(\operatorname{tg} \beta)$ foi maior no município de Londrina $\left(1,3 \times 10^{-3}\right)$ lote 8 no período de 300 dias de armazenamento, para a cultivar BRS 210, comparativamente com Mauá da Serra $\left(0,9 \times 10^{-3} \mathrm{e}\right.$ $\left.1,2 \times 10^{-3}\right)$, devido às condições de temperatura e umidade relativa do ar, pois quanto mais quente e úmido, maior a taxa de deterioração. Andreoli (2004), trabalhando com sementes de milho e soja, nas condições de Sete Lagoas, MG, e Brasília, DF, obteve resultados similares, o que 
corrobora com a equação de viabilidade de Ellis e Roberts (1980), assim como, Tang et al. (1999), trabalhando também com milho, mas com temperatura de armazenagem controlada de $30^{\circ} \mathrm{C}, 40^{\circ} \mathrm{C}$ e $50^{\circ} \mathrm{C}$.

$\mathrm{O}$ fato da taxa de deterioração ser maior em Londrina do que em Mauá da Serra, mostra que o valor da $\operatorname{tg} \beta$ equivale a $1 / \sigma$ da equação de Ellis e Roberts (1980). Nem o genótipo e nem a qualidade inicial da semente afetaram a declividade $(1 / \sigma$ e $\operatorname{tg} \beta)$ das curvas, somente a intercessão (Vi) da equação de viabilidade Eq. [9] foi afetada por tais fatores (Ellis e Roberts, 1980; Tang et al., 1999).

As altas correlações entre os valores observados e estimados pela equação $\left(r^{2}>0,95\right)$ revelam a alta confiabilidade do modelo em predizer a qualidade da semente no armazenamento convencional.

Para a cultivar IAPAR 78 (Figura 5a) e IPR 85 (Figura $5 \mathrm{~b}$ ), os oito lotes armazenados em Londrina foram significativos, ou seja, $\mathrm{r}^{2}=0.90,0.96,0,95$ e 0,94, 0,89, 0,95, 0,92 , respectivamente. A taxa de deterioração estimada pelo coeficiente angular $\operatorname{tg} \beta$ apresentou valores entre $0,7 \times 10^{3}$ a $1,2 \times 10^{-3}$ para a cultivar IAPAR 78 e entre $0,5 \times 10^{-3}$ a $0,9 \times 10^{-3}$ para a cultivar IPR 85 . Esses valores indicam que ocorreu maior deterioração no município de Londrina, PR, e o ângulo $\beta$ (a taxa de deterioração da semente) foi influenciado pelas condições climáticas locais. Popinigis (1985), obteve resultados similares trabalhando com semente de alfafa, submetidas à deterioração em diferentes temperaturas, com redução significativa do percentual de germinação, em temperaturas mais altas.

A germinação predita pela equação simplificada também foi comparada com a germinação observada, no período de 0 a 300 dias, em sementes de trigo para as cultivares BRS 208 (Figura 6a) e CD 104 (Figura 6b), As taxas de deterioração, estimadas pelo coeficiente angular $\operatorname{tg} \beta$, foram similares no município de Mauá da Serra $\left(0,4 \times 10^{-3}\right)$ e Londrina $\left(0,5 \times 10^{-3}\right)$, e o percentual de germinação ficou acima de $95 \%$, após um período de 300 dias em armazenamento convencional, nas duas localidades. As altas correlações entre os valores observados e estimados pela equação em Mauá da Serra $\left(r^{2}>0,82\right)$, e Londrina $\left(r^{2}>\right.$ 0,97 , revelam a alta confiabilidade do modelo em predizer a qualidade da semente no armazenamento convencional, validando o modelo prescrito por Tekrony et al. (1993), trabalhando com soja.
A equação simplificada estimou com acurácia a viabilidade das sementes de trigo, ao longo do período de armazenamento (180 e 300 dias) em Mauá da Serra e Londrina com uma variação de um ponto percentual (Figura 7). Isto demonstra que o genótipo não afetou a declividade da reta, ou seja, a taxa de deterioração da semente $(\operatorname{tg} \beta)$. Entretanto, Fabrizius et al. (1999), trabalhando com semente de soja, em armazenamento fechado (temperatura controlada) previu a taxa de deterioração para períodos de 430 dias e 800 dias com uma variação aproximada de dez pontos percentuais.

Assim, fica demonstrado que nas condições deste trabalho, os produtores de sementes poderão armazenar sementes de trigo por um período de 300 dias em Mauá da Serra e Londrina, com alto grau de confiabilidade, minimizando os custos advindos do processo de produção de sementes, devido a uma possível seleção prévia dos lotes, auxiliando os programas de controle de qualidade.

\section{CONSIDERAÇÕES FINAIS}

1. O modelo simplificado previu com acurácia mudanças na germinação e na a taxa de deterioração das sementes de trigo para as localidades de Mauá da Serra e Londrina.

2. As taxas de deterioração $(\operatorname{tg} \beta)$ das sementes de trigo variaram entre $0,4 \times 10^{-3}$ a $1,3 \times 10^{-3}$, e não foram afetadas pelo genótipo e pela germinação inicial da semente (Vi).

3. A taxa de deterioração $(\operatorname{tg} \beta)$ de semente de trigo em Londrina foi maior do que em Mauá da Serra.

\section{AGRADECIMENTOS}

Os autores agradecem à Empresa Sementes Mauá Ltda, Mauá da Serra-PR, por propiciarem as condições necessárias para a execução deste trabalho. A Marisa dos Santos Eumann do Departamento de Estatística, a equipe do Laboratório de Sementes da Embrapa-Soja, LondrinaPR e a Prof ${ }^{a}$. Dr ${ }^{a}$ Inês Cristina de Batista Fonseca da UEL, Londrina-PR. 


\section{REFERÊNCIAS}

ANDREOLI, C. The simplified equation to predict stability of mayze and soybean seeds. In: INTERNATIONAL CONFERENCE ON SEED SCIENCE AND TECHNOLOGY, 2., 1998, Guangzhou. Proceedings... Guangzhou: Zhongshan University, 1998. p.186198.

ANDREOLI, C. Simplificação da equação de viabilidade para predizer a longevidade da semente de milho e soja. Pesquisa Agropecuária Brasileira, Brasília, v.39, n.09, p.911-917, 2004.

BRASIL. Ministério da Agricultura e Reforma Agrária. Regras para Análise de Sementes. Brasília: SNDA/DNDV/CLAV, 1992. $365 \mathrm{p}$.

DELOUCHE, J.C.; BASKIN, C.C. Accelerated aging techniques for predicting the relative storability of seed lots. Seed Science and Technology, Zurique, v.1, n.2, p. 427-452, 1973.

ELLIS, R.H.; ROBERTS, E.H. Improved equations for the prediction of seed longevity. Annals of Botany, Londres, v. 45, p.13-30, 1980.

ELLIS, R.H.; ROBERTS,E.H. The qualification of aging and survival in orthodox seeds. Seed Science and Technology, Zurique, v.9, p.373-409, 1981.

ELLIS, R.H.: The viability equation, seed viability monographs, and practical advice on seed storage. Seed Science and Technology, Zurique, v.16, p.29-50, 1988.

ELLIS, R.H.; HONG, T.D.; ROBERTS, E.H. Moisture content and the longevity of seeds of Phaseolus vulgaris. Annals of Botany, Londres, v. 66, p.341-348,1990.

FABRIZIUS E.; TEKRONY, D.; EGLI, D.B.; RUCKER, M. Evolution of a viability model for predicting soybean seed germination during warehouse storage Crop Science, Alexandria, v.39, p.194-201, 1999.

FINNEY, D.J. Probit Analysis. 3 ed. Cambridge: Cambridge University Press, 1971.318p.
PARANÁ. Secretaria de Estado da Agricultura e do Abastecimento. Dados de Produção de Sementes: safra 2001/ 2002. Curitiba: SEAB, 2003. 57p.

POPINIGIS, F. Fisiologia da Semente. Brasília: AGIPLAN, 1985. 280p.

ROBERTS, E.H. The viability of cereal seed in relation to temperature and moisture. Annals of Botany, Londres, v.24, p.12$31,1960$.

ROBERTS, E.H. The viability of rice seed in relation to temperature, moisture content and gaseous environment. Annals of Botany, Londres, v.25, p.381-390, 1961.

ROBERTS, E.H.; ABDALLA, F.H. The influence of temperature, moisture, and oxygen on period of seed viability in barley, broad bens and peas. Annals of Botany, Londres, v.32, p.97-117, 1968.

ROBERTS, E.H. Storage environment and the control of viability. In: ROBERTS, E. H. (Ed.). Viability of seed. Syracuse: Syracuse University Press, 1972. p.14-58.

ROBERTS, E.H. Predicting the storage life of seeds. Seed Science and Technology, Zurique, v.1, n.3, p.499-514, 1973.

ROBERTS, E.H. Quantifying seed deterioration. In: McDONALD JR, M.B; NELSON, C.J. (Ed.).Physiology of seed deterioration. Madison: Crop Science Society of America, 1986. p.101-123. (Special Publication,11).

ROBERTS, E.H.; ELLIS, R.H. Water and seed survival. Annals of Botany, Londres, v 63, p.39-52, 1989.

TANG, S.; TEKRONY, D.M.; EGLI, D.B.; CORNELIUS, P.L.; RUCHER, M. Survival characteristics of corn seed during storage: I. Normal distribution of seed survival. Crop Science, Alexandria, v.39, p.1394-1400, 1999.

TANG, S.; TEKRONY, D.M., EGLI, D.B.; CORNELIUS, P.L. An alternative model to predict corn seed deterioration during storage. Crop Science, Alexandria, v.40, p.463-470, 2000.

TEKRONY,D.M.; NELSON, C.; EGLI, D.B.; EGLI, G.M. Predicting soybean seed deterioration during warehouse storage. Seed Science and Technology, Zurique, v.21, p.127-137, 1993.

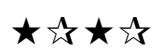

